\title{
Probing Orbital Structure of Polyatomic Molecules by High-Order Harmonic Generation
}

\author{
R. Torres, ${ }^{1}$ N. Kajumba, ${ }^{1}$ Jonathan G. Underwood, ${ }^{2}$ J. S. Robinson, ${ }^{1}$ S. Baker, ${ }^{1}$ J. W. G. Tisch, ${ }^{1}$ R. de Nalda,${ }^{3}$ W. A. Bryan, ${ }^{4}$ \\ R. Velotta, ${ }^{5}$ C. Altucci, ${ }^{5}$ I. C. E. Turcu, ${ }^{4}$ and J. P. Marangos ${ }^{1}$ \\ ${ }^{1}$ Blackett Laboratory, Imperial College London, London SW7 2BW, United Kingdom \\ ${ }^{2}$ Department of Physics and Astronomy, The Open University, Walton Hall, Milton Keynes MK7 6AA, United Kingdom \\ ${ }^{3}$ Instituto de Química-Física Rocasolano, CSIC, 28006 Madrid, Spain \\ ${ }^{4}$ Central Laser Facility, CCLRC Rutherford Appleton Laboratory, Chilton, Didcot, Oxon OX11 OQX, United Kingdom \\ ${ }^{5}$ CNISM and Dipartimento di Scienze Fisiche, Università di Napoli “Federico II," Napoli, Italy
}

(Received 4 December 2006; published 17 May 2007; corrected 29 May 2007)

\begin{abstract}
The effects of electronic structure and symmetry are observed in laser driven high-order harmonic generation for laser aligned conjugated polyatomic molecular systems. The dependence of the harmonic yield on the angle between the molecular axis and the polarization of the driving laser field is seen to contain the fingerprint of the highest occupied molecular orbitals in acetylene and allene, a good quantitative agreement with calculations employing the strong field approximation was found. These measurements support the extension of the recently proposed molecular orbital imaging techniques beyond simple diatomic molecules to larger molecular systems.
\end{abstract}

PACS numbers: $33.80 . \mathrm{Rv}, 42.65 . \mathrm{Ky}, 42.65 . \mathrm{Re}$

High-order harmonic generation (HHG) in atomic gases by high intensity laser fields has proven a fruitful method for producing coherent extreme ultraviolet beams and attosecond pulses [1-3]. In molecules, HHG presents a rich set of new physical phenomena, as both the ionization and electron recombination steps of HHG are dependent on the particular symmetry of the highest occupied molecular orbital (HOMO) and its orientation with respect to the laser field [4-9]. Because of this orientation dependence, HHG in molecules can provide a unique time resolved probe of the electronic orbital structure. Recent work has shown that the HHG spectrum from aligned molecular samples can be used to determine the molecular structure for small molecules such as $\mathrm{N}_{2}$ and $\mathrm{CO}_{2}$ [10-15]. So far these studies have been confined to simple diatomic and triatomic linear molecules. The present work extends this approach to significantly more complex polyatomic molecules, and we find that the angular dependence of the HHG signal can be used to map more complex electronic orbitals.

In order to perform a full tomographic reconstruction of the molecular orbital, a high degree of spatial alignment of the molecules is required; furthermore, a large enough number of harmonic orders must be produced in order to provide a complete characterization of the orbital in momentum space. The structure retrieval is then possible by either the examination of distinct interference signatures in the spectrum [10-12] or tomographic reconstruction $[13,14]$ or the iterative optimization of the wave functions to best match the measured spectrum [15]. The mathematical steps in structural retrieval from HHG are based upon the strong field approximation (SFA). The SFA is a single active electron model and, additionally, the influence of the laser field upon the bound electronic states, and the influence of the Coulomb potential upon the continuum electron states are both neglected. HHG in the strong field limit can be seen as a three step process [16]: (i) ionization; (ii) acceleration in the laser field; (iii) recombination back into the bound electronic state. It is known that multielectron processes become significant with increasing molecular size [17] as, for example, the polarization of the ionic core by the laser field modifies the strong field processes. Furthermore, the limitations of the SFA due to the lack of translational and gauge invariance [18] are expected to be more significant for extended molecular systems. It is therefore critical that an assessment of the applicability of the SFA to larger molecules is made, since this approach underpins current approaches to structural retrieval from HHG. The experiments reported here address precisely this issue.

For small molecules, such as $\mathrm{N}_{2}$ and $\mathrm{CO}_{2}$, good agreement between experiment and SFA calculations has been obtained for HHG from aligned molecules [8,19]. For larger molecules (ethylene and benzene), while good agreement is found between experiment and SFA calculations at least for the ionization rates of randomly oriented gas samples [20], it remains to be seen whether the SFA provides a reliable description of HHG from aligned molecules that includes not only the ionization processes but also the acceleration and recombination steps. Furthermore, such HHG experiments with aligned molecules allow the molecular frame angular dependence of these processes to be examined in detail. These details are not available from experiments on randomly oriented targets which suffer an averaging over all molecular orientations.

The measurements reported here are the first measurements of HHG from aligned organic molecules and are in good agreement with the results of an SFA calculation, confirming the viability of using the SFA for connecting the molecular structure to the HHG signal in a wider class of molecules. In the present work we use the conjugated organic molecules acetylene $(\mathrm{HC} \equiv \mathrm{CH})$ and allene 
$\left(\mathrm{H}_{2} \mathrm{C}=\mathrm{C}=\mathrm{CH}_{2}\right)$ as prototypes for larger polyatomic molecules. In both molecules the HOMO is dominated by bonding $\pi_{u}$ orbitals between the carbon atoms [see Figs. 1(a) and 1(b)], though the allene molecule has additional contributions to the HOMO from $\mathrm{C}-\mathrm{H}$ bonds.

The experiment was performed using the $10 \mathrm{~Hz}$ ASTRA Laser Facility at the Rutherford Appleton Laboratory, U.K. This system provides laser pulses of $\sim 10 \mathrm{fs}$ duration and $\sim 0.5 \mathrm{~mJ}$ at $800 \mathrm{~nm}$ after a hollow fiber pulse compressor. A schematic of the experimental setup is shown in Fig. 1(c). Approximately $80 \%$ of the beam energy was split off before the hollow fiber and sent through a delay line in order to produce an aligning pulse correctly synchronized with the harmonic generating field. These beams were recombined with a normal incidence beam splitter and propagated collinearly into the interaction chamber.

The beams were focused by a $40 \mathrm{~cm}$ focal length off-axis parabolic mirror into a pulsed gas jet. The stagnation pressure of the gases was $2 \mathrm{bar}$, and the nozzle diameter was $100 \mu \mathrm{m}$. The temperature of the gas in the interaction region was estimated to be less that $100 \mathrm{~K}$, low enough to ensure a high degree of molecular alignment. The alignment beam had an energy of $200 \mu \mathrm{J}$ and a pulse duration of $60 \mathrm{fs}$, giving an estimated on target intensity of $\sim 3 \times$ $10^{13} \mathrm{~W} / \mathrm{cm}^{2}$, which was insufficient to cause any detectable HHG. The harmonic generating beam had an energy of $300 \mu \mathrm{J}$ and $14 \mathrm{fs}$ duration, providing an on target
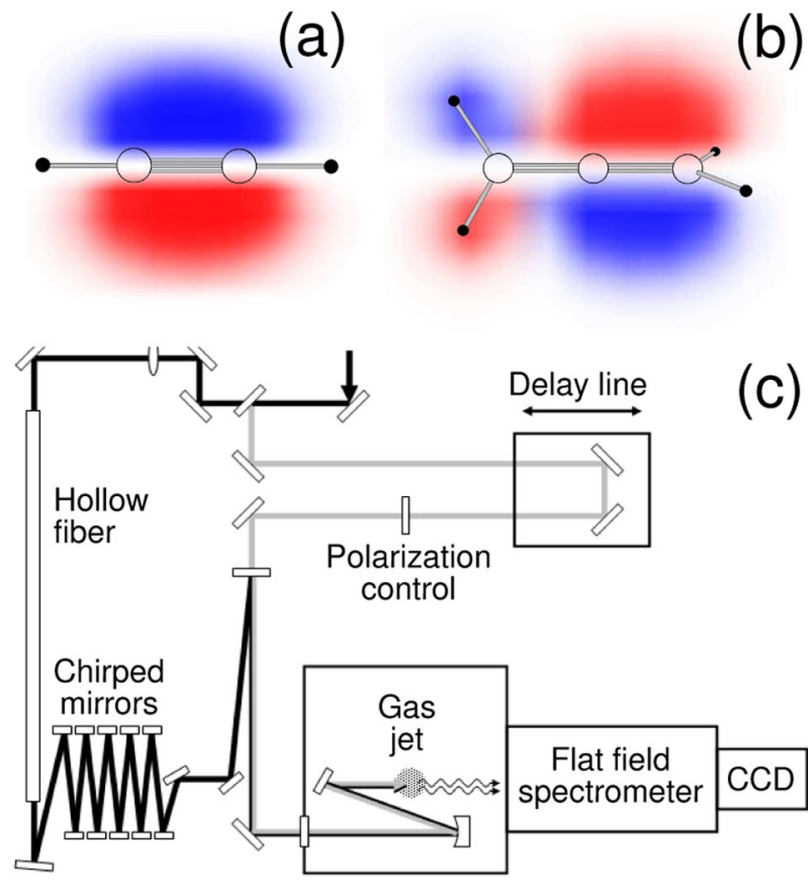

FIG. 1 (color online). (a),(b) Representation of one of the two degenerate orbitals comprising the HOMO in acetylene and allene, respectively, based upon a calculation with GAMESS-UK. Different colors indicate different signs of the wave function. (c) Schematic layout of the experiment. The gray and black lines represent the aligning and harmonic generating beams, respectively. intensity of $\sim 1.5 \times 10^{14} \mathrm{~W} / \mathrm{cm}^{2}$. The short duration of the harmonic generating pulse is very important for accurate structural retrieval since it prevents a significant realignment of the molecules at the time of harmonic emission and avoids saturation of the ionization. A halfwave plate in the aligning beam controlled the angle $\Theta$ between the aligning and harmonic generating fields. The harmonics produced in the gas jet were spatially dispersed by a flat field spectrometer and detected with a microchannel plate detector fitted with a phosphor screen. A CCD camera was used to image the phosphor screen.

Harmonics from the 17th to the 27th order were detected in acetylene and allene. The harmonic spectra were recorded for different time delays between the aligning and driving pulses while maintaining their polarizations parallel. Spectra were also recorded in the absence of an aligning field under otherwise identical conditions; when correctly calibrated, these spectra were used to extract the signal ratio between the aligned and isotropic samples.

The signal of each harmonic shows a modulation as a function of the time delay that corresponds to different degrees of alignment. The degree of alignment may be represented by the expectation value $\left\langle\cos ^{2} \theta\right\rangle$, where $\theta$ is the angle between the molecular axis (i.e., the symmetry axis of the molecule) and the aligning field polarization. Acetylene and allene, being linear and symmetric top molecules, respectively, exhibit regular rotational energy level spacings, which results in regular revivals of the molecular axis alignment after the aligning pulse [21]. The modulation in the harmonic yield observed was anticorrelated with the calculated evolution of $\left\langle\cos ^{2} \theta\right\rangle$ for all harmonic orders in both molecules (Fig. 2), indicating a suppression in the harmonic yield when the molecules are aligned with their axes parallel to the driving field polarization. The incoherent alignment $[22,23]$ is also evidenced as a constant signal suppression between the revivals.

Setting the delay at times corresponding to maximum alignment (along the laser field) or maximum antialignment (located preferentially in the plane perpendicular to the aligning field), the signal was recorded as a function of
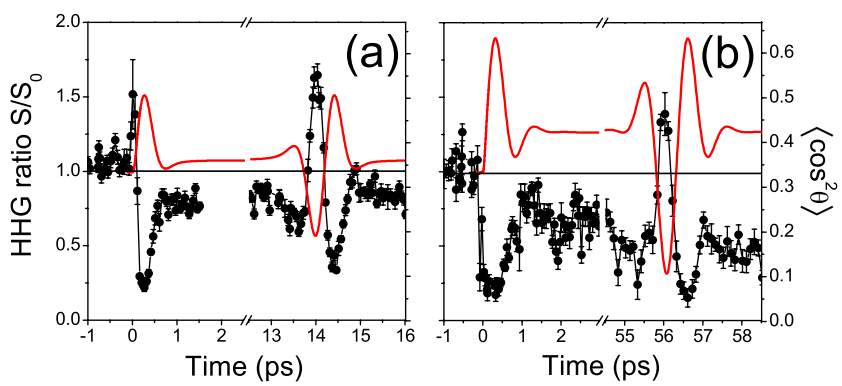

FIG. 2 (color online). Ratio of harmonic intensity between aligned and nonaligned samples as a function of time delay between the aligning and harmonic generating pulses for (a) the 19th harmonic in acetylene and (b) the 19th harmonic in allene. The gray (red) solid curves show the calculated evolution of $\left\langle\cos ^{2} \theta\right\rangle$. 
the angle $\Theta$ between the aligning and probe field polarization directions. The angular dependence of the signal ratio taken at the time of maximum alignment (full revival) in acetylene $\left(t_{0}=14.38 \mathrm{ps}\right)$ and allene $\left(t_{0}=56.55 \mathrm{ps}\right)$ are plotted in the left column of Figs. 3 and 4 . For all harmonic orders in both molecules, the harmonic yield is suppressed when the molecules are predominantly parallel to the field and increases with the angle. At the points of maximum antialignment in acetylene and allene $\left(t_{0}=14.00 \mathrm{ps}\right.$ and $t_{0}=56.00 \mathrm{ps}$, respectively) the signal ratios display the opposite trend with $\Theta$ to those taken at the full revival, as expected (right column of Figs. 3 and 4). In acetylene the maximum is reached at $\Theta=90^{\circ}$; in contrast, for allene the signal ratio shows a significantly different behavior with the maximum shifted to $\Theta<90^{\circ}$. This is a direct consequence of the different molecular orbital structure.

The experimental results were simulated in the following way: First, the harmonic spectra were calculated using the approach described by Lewenstein et al. [24] following the assumptions of the SFA and using the saddle-point approximation. The depletion of the ground state was also ignored since the laser intensities employed here kept the ionization far from saturation. Assuming a driving field linearly polarized in the $z$ direction, the whole information about the molecule is contained in the transition dipole matrix element from the HOMO state $\psi_{0}(\mathbf{r})$ to a continuum state expressed as a plane wave of momentum $p: d_{z}[p]=\left\langle p|z| \psi_{0}(\mathbf{r})\right\rangle$.

The HOMO orbitals $\psi_{0}(\mathbf{r})$ are calculated using the GAMESS-UK package [25] [Figs. 1(a) and 1(b)]. Keeping

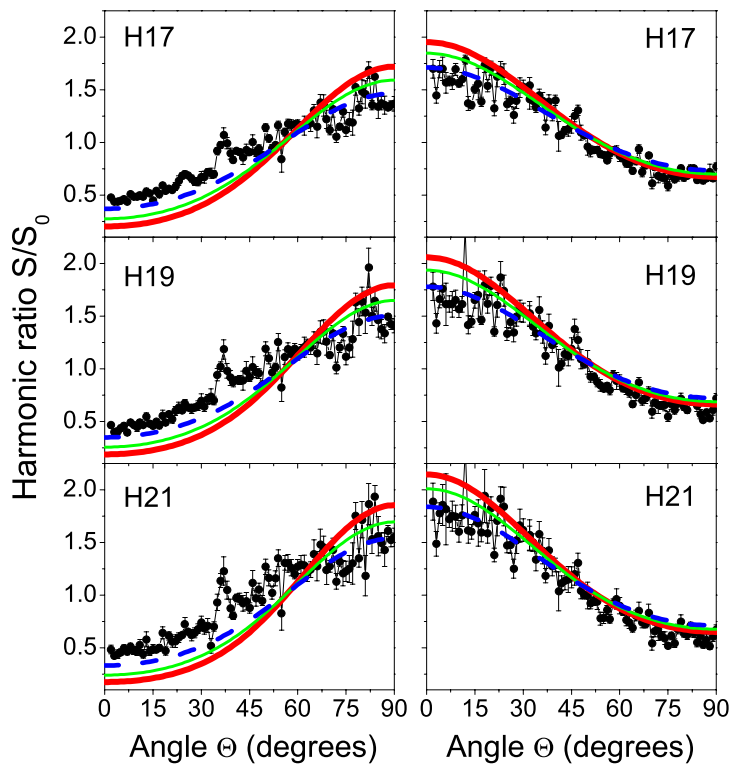

FIG. 3 (color online). Harmonic ratio between aligned and nonaligned acetylene molecules as a function of polarization angle between aligning and driving beams, measured at the alignment peak ( $\left.t_{0}=14.38 \mathrm{ps}\right)$ (left column) and the antialignment peak ( $\left.t_{0}=14.00 \mathrm{ps}\right)$ (right column). The red thick solid, green thin solid, and blue dashed curves show the calculated ratio in a sample at $20,50 \mathrm{~K}$, and $100 \mathrm{~K}$, respectively. the electric field fixed in space, the different orientations of the molecules are introduced by applying the Euler operator $\tilde{\mathbf{R}}_{\phi \theta \chi}$ on the spatial coordinates of the orbitals. As the laser is linearly polarized, the dipole moment is independent of the angle of rotation around the polarization vector $\phi$. Thus we get $d_{z}[p ; \theta, \chi]=\left\langle p|z| \psi_{0}\left(\tilde{\mathbf{R}}_{\phi \theta \chi} \mathbf{r}\right)\right\rangle$ [26]. Introducing this expression in the Lewenstein model, we obtain an angle dependent harmonic spectrum $S(\omega ; \theta, \chi)$. The dependence on $\chi$ must be averaged, as only the polar angle $\theta$ is controlled in the experiments. In this calculation we consider only one component of the doubly degenerate HOMO of the molecules, as any other linear combination of the two components must give the same results averaged over $\chi$ provided they are orthogonal [27].

The angular dependence of the amplitude of the $n$th harmonic, $a_{n}(\theta)$, is calculated integrating the amplitude spectrum $S(\omega ; \theta)$ between $\omega_{n-1 / 2}$ and $\omega_{n+1 / 2}$, where $\omega_{n}$ is the frequency of the harmonic. The angular dependence of the phase is calculated as $p_{n}(\theta)=\arg \left[S\left(\omega_{n} ; \theta\right)\right]$.

In order to compare the calculations with the experimental results we must take into account the distribution of molecular orientations produced by the aligning field at a time $t_{0}$ after the aligning pulse. The experimental angular dependence of the $n$th harmonic yield measured at a time $t_{0}$ after the aligning pulse is

$$
S_{n}\left(t_{0} ; \Theta\right)=\left|\int_{0}^{\pi} a_{n}(\theta) \exp \left[i p_{n}(\theta)\right] \sigma_{\Theta}\left(t_{0} ; \theta\right) d \theta\right|^{2},
$$

where $\sigma_{\Theta}\left(t_{0} ; \theta\right)$ is the polar angle distribution of the mo-

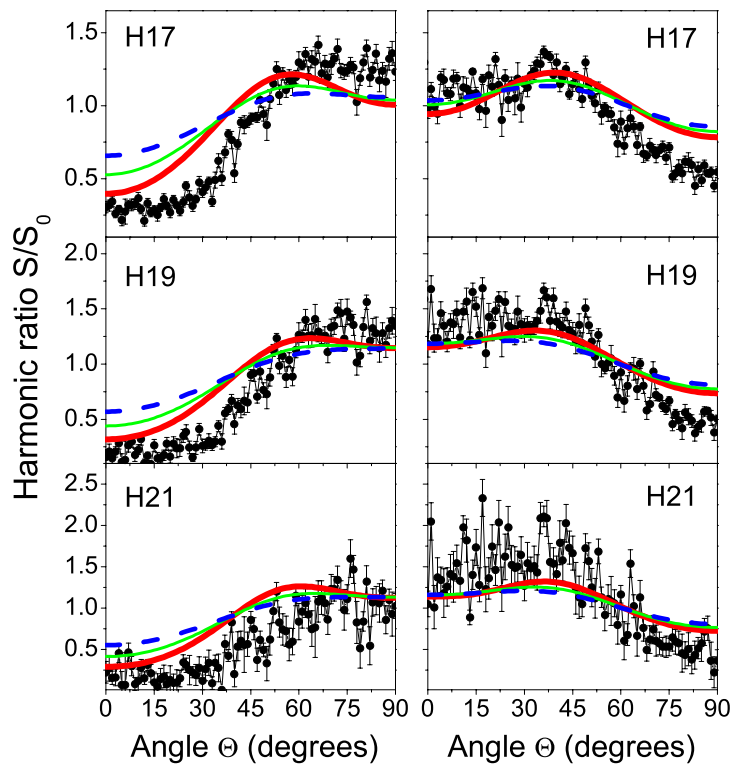

FIG. 4 (color online). Harmonic ratio between aligned and nonaligned allene molecules as a function of polarization angle between aligning and driving beams, measured at the alignment

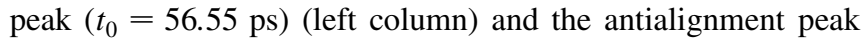
$\left(t_{0}=56.00 \mathrm{ps}\right)$ (right column). The red thick solid, green thin solid, and blue dashed curves show the calculated ratio in a sample at 20, 50, and $100 \mathrm{~K}$, respectively. 
lecular ensemble at time $t_{0}$, probed at an angle $\Theta$ from the aligning field direction, calculated following the procedure described in [28]. Finally, Eq. (1) is normalized by the signal calculated from an isotropic ensemble, $S_{0 n}$.

Because of the uncertainty in the rotational temperature of the samples the calculations have been made for three different temperatures. The calculated data for 20,50, and $100 \mathrm{~K}$ are plotted in Figs. 3 and 4 as red thick solid, green thin solid, and blue dashed lines, respectively, and they show a good agreement with the experimental results. The modulation of the calculated signal with the sampling angle $\Theta$ depends upon the degree of alignment in the molecular ensemble, as expected. The aligning pulse intensity used in the calculations was $3 \times 10^{13} \mathrm{~W} / \mathrm{cm}^{2}$, and the pulse duration was taken to be $60 \mathrm{fs}$; the probe pulse was assumed to have an intensity of $1.5 \times 10^{14} \mathrm{~W} / \mathrm{cm}^{2}$ and 14 fs duration.

These results illustrate how the angular dependence of the harmonic yield is related to the shape of the orbitals. The signal suppression around $0^{\circ}$ is due to the nodal plane in the orbitals of acetylene and allene, which contain their molecular axes, and the signal for larger angles increases as the electron density in the direction of the field grows larger. In the case of acetylene, the maximum occurs in the direction perpendicular to the molecular axis. This behavior is characteristic of a $\pi_{u}$ orbital. In allene, while the general trend shows the same pattern as acetylene in accordance with the same $\pi_{u}$ structure that dominates the HOMO, we see a clear difference in the experimental signal at large sampling angles that is reproduced by the calculations. These results agree with the interpretation of previous measurements of $\mathrm{HHG}$ in $\mathrm{N}_{2}$ and $\mathrm{O}_{2}$ where the angular dependence has been found to be characteristic of their HOMO structure $\left(\sigma_{g}\right.$ and $\pi_{g}$, respectively) $[6,8,9]$. However, this is clear evidence that the effect of subtler structural details of the molecular orbital can be observed in the angular dependence of the harmonic signal.

The range of harmonics explored here is insufficient to carry out a complete tomographic reconstruction of the orbitals. Nevertheless, the present measurements represent a step forward in the generalization of the HHG imaging technique to more complex molecules by showing that the SFA can still be adequately employed, and that the measured angle dependent spectra carry information on the structure and symmetry of the molecular orbital. This also implies that, for these molecules at least, a calculation using only a single electron wave function is sufficient to capture the main features of the angular dependence of HHG.

We gratefully acknowledge the support of the staff of the Central Laser Facility at Rutherford Appleton Laboratory. R. T. also acknowledges the Spanish Department of the State of Education and Universities, and the European Social Fund for financial support. The experiment time was granted by the CCLRC (Experiment No. A2P2/05) in the Target Area One of the Astra Laser Facility. This work has been supported by EPSRC and RC U.K.

[1] K. Burnett, V. C. Reed, and P. L. Knight, J. Phys. B 26, 561 (1993).

[2] P. Antoine, A. L'Huillier, and M. Lewenstein, Phys. Rev. Lett. 77, 1234 (1996).

[3] M. Drescher et al., Science 291, 1923 (2001); P. M. Paul et al., Science 292, 1689 (2001).

[4] R. Velotta, N. Hay, M. B. Mason, M. Castillejo, and J. P. Marangos, Phys. Rev. Lett. 87, 183901 (2001).

[5] B. Shan, X.-M. Tong, Z. Zhao, Z. Chang, and C. D. Lin, Phys. Rev. A 66, 061401(R) (2002).

[6] B. Shan, S. Ghimire, and Z. Chang, Phys. Rev. A 69, 021404(R) (2004).

[7] R. de Nalda et al., Phys. Rev. A 69, 031804(R) (2004).

[8] X. X. Zhou, X.-M. Tong, Z. X. Zhao, and C. D. Lin, Phys. Rev. A 71, 061801(R) (2005).

[9] C. B. Madsen and L. B. Madsen, Phys. Rev. A 74, 023403 (2006).

[10] M. Lein, N. Hay, R. Velotta, J.P. Marangos, and P. L. Knight, Phys. Rev. Lett. 88, 183903 (2002).

[11] T. Kanai, S. Minemoto, and H. Sakai, Nature (London) 435, 470 (2005).

[12] C. Vozzi et al., Phys. Rev. Lett. 95, 153902 (2005).

[13] J. Itatani et al., Nature (London) 432, 867 (2004).

[14] S. Patchkovskii, Z. Zhao, T. Brabec, and D. M. Villeneuve, Phys. Rev. Lett. 97, 123003 (2006).

[15] V.-H. Le, A.-T. Le, R.-H. Xie, and C.D. Lin, arXiv:physics/0701250v1.

[16] P. B. Corkum, Phys. Rev. Lett. 71, 1994 (1993).

[17] M. Smits, C.A. de Lange, A. Stolow, and D.M. Rayner, Phys. Rev. Lett. 93, 203402 (2004); 93, 213003 (2004).

[18] C. C. Chirilă and M. Lein, Phys. Rev. A 73, 023410 (2006).

[19] J. Itatani et al., Phys. Rev. Lett. 94, 123902 (2005).

[20] T.K. Kjeldsen, C.Z. Bisgaard, L.B. Madsen, and H. Stapelfeldt, Phys. Rev. A 71, 013418 (2005).

[21] H. Stapelfeldt and T. Seideman, Rev. Mod. Phys. 75, 543 (2003).

[22] F. Rosca-Pruna and M. J. J. Vrakking, J. Chem. Phys. 116, 6567 (2002)

[23] P. W. Dooley et al., Phys. Rev. A 68, 023406 (2003).

[24] M. Lewenstein, Ph. Balcou, M. Yu. Ivanov, A. L'Huillier, and P. B. Corkum, Phys. Rev. A 49, 2117 (1994).

[25] M. W. Schmidt et al., J. Comput. Chem. 14, 1347 (1993).

[26] Note that $\Theta$ is the angle between the driving and aligning fields, while $\theta$ is the angle between the driving field and molecular axis, so for each value of $\Theta$ there is a distribution of molecular orientations $\sigma_{\Theta}(\theta)$.

[27] T. K. Kjeldsen, C.Z. Bisgaard, L.B. Madsen, and H. Stapelfeldt, Phys. Rev. A 68, 063407 (2003).

[28] R. Torres, R. de Nalda, and J. P. Marangos, Phys. Rev. A 72, 023420 (2005). 